\title{
SOLUTION OF A ONE-DIMENSIONAL DIFFUSION-REACTION MODEL WITH SPATIAL ASYMMETRY
}

\author{
Haye Hinrichsen ${ }^{\natural}$, Klaus Krebs ${ }^{\star}$ and Ingo Peschel ${ }^{\diamond}$ \\ $\diamond$ Freie Universität Berlin, Fachbereich Physik \\ Arnimallee 14, D-14195 Berlin, Germany \\ * Universität Bonn, Physikalisches Institut \\ Nußallee 12, D-53115 Bonn, Germany
}

\begin{abstract}
We study classical particles on the sites of an open chain which diffuse, coagulate and decoagulate preferentially in one direction. The master equation is expressed in terms of a spin one-half Hamiltonian $H$ and the model is shown to be completely solvable if all processes have the same asymmetry. The relaxational spectrum is obtained directly from $H$ and via the equations of motion for strings of empty sites. The structure and the solvability of these equations are investigated in the general case. Two phases are shown to exist for small and large asymmetry, respectively, which differ in their stationary properties.
\end{abstract}

Key words: Reaction-diffusion systems, non-equilibrium statistical mechanics, coagulation model, integrability, spatial asymmetry

PACS numbers: $05.40 .+\mathrm{j}$, 05.70.Ln, 82.20.Mj

\footnotetext{
${ }^{1}$ Submitted to Zeitschrift für Physik

${ }^{2}$ Present address : Dept. of Physics of Complex Systems, Weizmann Institute of Science, Rehovot 76100, Israel
} 


\section{Introduction}

The study of exactly solvable models has been an important activity in equilibrium statistical physics and it is equally desirable to find such models in the field of non-equilibrium phenomena. In this context it has proved very useful to exploit the formal analogy between the master equation for classical stochastic systems and the quantum-mechanical Schrödinger equation. For example, by relating the one-dimensional kinetic Ising model to a quantum spin chain [1, 2, 3, 4] it becomes obvious why the system is solvable with Glauber's [5] choice of the rates. Similarly, the hopping of classical particles with hard-core repulsion on a lattice can be formulated as a spin problem, namely the ferromagnetic Heisenberg model [6, 7], and in one dimension this is again a solvable model.

In recent work, this approach has been extended to more general situations. They include diffusion with a preferred direction as well as reactions between particles or coupling to external reservoirs [8, 9, 10, 11, 12, 13, 14]. The spin of the resulting quantum models is $s+1$ if $s$ types of particles are involved. Fermionic or bosonic representations have also been used, for example for models of self-organized criticality [15, 16]. In general, the inclusion of more processes makes the time evolution operator more complicated. On the other hand, by tuning the various rates one may also achieve simplifications which make the problem integrable in some cases.

One of the systems where this happens is the coagulation model in which particles hop on a one-dimensional lattice and, in addition, can merge and separate again with certain probabilities. This model was introduced and treated in a continuum approximation by Doering et.al. [17, 18, 19, 20. It was found later that it is exactly solvable on a lattice if the rates for hopping and for coagulation are equal [21]. The time evolution operator can then be brought into the form of the Hamiltonian for a spin one-half $X Y$ chain with a $Z$ field. It is the dual of the operator for the Glauber model mentioned above and can be diagonalized in terms of fermions. The spectrum has a gap determined by the decoagulation rate. If this rate vanishes, one finds an algebraic decay of the concentration with exponent minus one-half. The model can then be mapped onto a pair annihilation model [21] and there is also an interesting physical realization in terms of excitons in the quasi one-dimensional substance TMMC [22].

In treating this model, it turned out that physical quantities like the density are most easily calculated from the probabilities to find empty intervals of arbitrary length ("holes"). Their equations of motion form closed sets and can be solved for a ring as well as for a chain. They have been used also in related models [23]. An intriguing aspect is that these closed sets even exist if the creation of particles is allowed [24]. The relaxational spectrum of the hole functions then has the form of the Wannier-Stark ladder found for lattice electrons in a homogeneous electric field [25, 26]. However, no fermionic solution is possible in this case and the question of complete integrability has remained open so far.

In the present article we study the properties of the coagulation model for the case of a preferred direction. The geometry will be the open chain where an interplay between the boundaries and the spatial asymmetry occurs. Our aim is to pursue the question of integrability further, and also to find the basic physical features of the model. We focus on the case where all processes have the same asymmetry and the rates for hopping and coagulation are chosen equal. Then the model contains two free parameters. We show that, as for symmetric 
rates, the spin operator describing the time evolution can be brought into a form where it is quadratic in fermions. Thus one finds complete integrability also in this case. However, in contrast to simple hopping or symmetric coagulation the problem has no quantum group symmetry. This can be related to particular boundary terms which correspond to fields in the magnetic language and lead to special selection rules. There is, however, a discrete $S_{3}$-symmetry which shows up in the multiplicity of the relaxational modes.

We also consider the hole equations, both in the one-hole sector and in general. Following an approach by Bedeaux et.al. [27] the different levels in the hierarchy can be decoupled and a complete solution achieved. By considering more general rates and processes, it is seen that this procedure only works in the free fermion case. This is also what the Reshetikhin criterion for integrability 28] gives. Thus the other cases where the one-hole equations can be solved, correspond to partially integrable systems. Such a situation occurs also in kinetic spin models [3, 29] and in reaction-diffusion models where for particular rates the equations for the density correlations form closed sets 30.

The physical properties of the model are also interesting. In the stationary state the density is inhomogeneous and determined by a competition between asymmetry and decoagulation. If the latter is small, the density is finite only near one boundary. In the opposite case, it is constant in the bulk of the system with some additional boundary effects. In between, a transition takes place, where the profile becomes linear over the major part of the system. These different regimes are also reflected in the form of the relaxation spectrum. In general, one finds a gap but at the transition it vanishes and one has slow modes as in symmetric diffusion. The phenomenon may be viewed as another example of a boundaryinduced transition $13,31,32,33$.

We shall present the material as follows. In section 2 we introduce the model and the spin formalism used in its treatment. The time evolution operator is derived and brought into convenient forms in section 3. In section 4 its spectrum is found by diagonalization via fermions. Section 5 deals with the equations of motion in the one-hole sector and in section 6 the $n$-hole problem is treated via cumulant-like functions. From these results, density profile and correlation functions in the steady state are obtained as special cases and discussed in section 7 . Finally, section 8 contains a summary and concluding remarks.

\section{$2 \quad$ Model}

We consider classical particles on a chain of $L$ sites, each of which can either be empty or occupied with at most one particle. The dynamics of the system consists of transitions which involve only the configurations of neighbouring sites. As discussed previously [24], one can then distinguish altogether 12 different processes, namely hopping, coagulation and decoagulation as well as birth and death of single particles and of pairs. Each process is described by a rate constant and, in the absence of detailed balance, all these constants are independent.

In the bulk of the paper we will only consider the first three processes which are shown below together with their rates: 

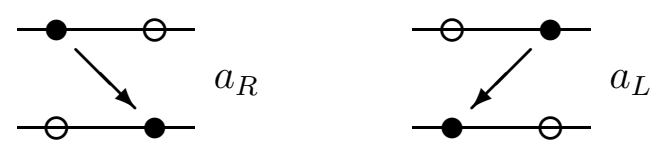

Hopping
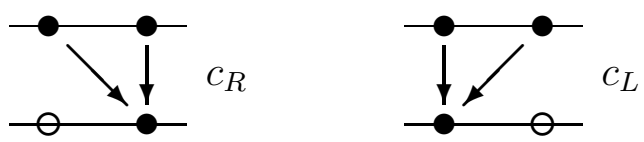

Coagulation
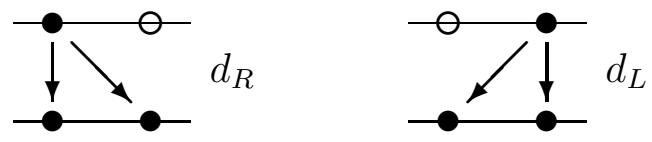

Decoagulation

The left-right asymmetry which we assume could result from external fields. We will use a spin language to describe the system. The state of site $n$ is specified by a variable $\sigma_{n}= \pm 1$ such that $\sigma_{n}=+1(-1)$ if the site is occupied (empty). A configuration of the total system is denoted by $\underline{\sigma}=\left\{\sigma_{1}, \sigma_{2}, \ldots \sigma_{L}\right\}$. The probability $P(\underline{\sigma}, t)$ to find configuration $\underline{\sigma}$ at time $t$ then obeys the master equation

$$
\frac{\partial}{\partial t} P(\underline{\sigma}, t)=-\sum_{\underline{\sigma}^{\prime}} P(\underline{\sigma}, t) w\left(\underline{\sigma} \rightarrow \underline{\sigma}^{\prime}\right)+\sum_{\underline{\sigma}^{\prime}} P\left(\underline{\sigma}^{\prime}, t\right) w\left(\underline{\sigma}^{\prime} \rightarrow \underline{\sigma}\right)
$$

where the two terms on the right represent the loss and the gain processes, respectively. The transition probabilities $w\left(\underline{\sigma} \rightarrow \underline{\sigma}^{\prime}\right)$ are sums of contributions from the various nearest neighbour processes.

To formulate the problem in quantum-mechanical terms [1, 34] one uses a ket vector notation in the $2^{L}$-dimensional state space, writing

$$
|P(t)\rangle=\sum_{\underline{\sigma}} P(\underline{\sigma}, t)|\underline{\sigma}\rangle .
$$

The master equation then takes the form

$$
\frac{\partial}{\partial t}|P(t)\rangle=-H|P(t)\rangle
$$

from which the analogy with the Schrödinger equation is obvious. The time evolution operator $H$ will therefore be called Hamiltonian in the following. However, since $|P(t)\rangle$ is already the probability, expectation values differ from the quantum-mechanical ones. For a quantity $A(\underline{\sigma})$ one has

$$
\langle A(t)\rangle=\sum_{\underline{\sigma}} A(\underline{\sigma}) P(\underline{\sigma}, t)=\langle 0|A| P(t)\rangle
$$

with the bra vector

$$
\langle 0|=\sum_{\underline{\sigma}}\langle\underline{\sigma}| .
$$

The operator $H$ is the sum of nearest-neighbour terms

$$
H=\sum_{n=1}^{L-1} H_{n, n+1}
$$


and the $(4 \times 4)$ matrix $H_{n, n+1}$ can be written down easily [24]. In a basis of states $(|--\rangle, \mid-$ $+\rangle,|+-\rangle,|++\rangle)$ the result is, including birth processes (rates $b_{R}, b_{L}$ ) and pair creation (rate $c$ ) for further reference

$$
H_{n, n+1}=\left(\begin{array}{cccc}
c+b_{L}+b_{R} & 0 & 0 & 0 \\
-b_{R} & a_{L}+d_{L} & -a_{R} & -c_{R} \\
-b_{L} & -a_{L} & a_{R}+d_{R} & -c_{L} \\
-c & -d_{L} & -d_{R} & c_{L}+c_{R}
\end{array}\right)
$$

Due to probability conservation, the elements in the columns sum to zero. $H_{n, n+1}$ and therefore the total operator $H$ is non-hermitean.

In the next sections we will consider the case of no birth and pair creation $\left(b_{R}=b_{L}=\right.$ $c=0)$, equal rates for hopping and coagulation $\left(c_{R}=a_{R}, c_{L}=a_{L}\right)$ and equal asymmetry for all processes. Up to a common constant which we set equal one, the rates can then be written

$$
a_{R}=\frac{1}{q}, \quad a_{L}=q, \quad d_{R}=\frac{\Delta}{q}, \quad d_{L}=\Delta q .
$$

Thus the parameter $q$ as usual describes the asymmetry, while $\Delta$ gives the ratio of decoagulation to coagulation rates and therefore governs the (average) density.

\section{Time evolution operator}

Starting e.g. from Eq. (2.7), the Hamiltonian $H$ can be expressed in terms of Pauli-matrices $\sigma_{i}^{\alpha}(\alpha=x, y, z)$. It is the sum of diffusion and coagulation contributions

$$
H=H_{D}+H_{C},
$$

where $H_{D}$ is given by

$$
\begin{aligned}
H_{D}=-\frac{1}{4} \sum_{i=1}^{L-1}\{ & q^{-1} \sigma_{i}^{-} \sigma_{i+1}^{+}+q \sigma_{i}^{+} \sigma_{i+1}^{-}+\left(q+q^{-1}\right) \sigma_{i}^{z} \sigma_{i+1}^{z} \\
& \left.+\left(q-q^{-1}\right)\left(\sigma_{i}^{z}-\sigma_{i+1}^{z}\right)-\left(q+q^{-1}\right)\right\} .
\end{aligned}
$$

The standard $U_{q}[S U(2)]$-symmetric form of $H_{D}$ is then obtained by a position-dependent rescaling of $\sigma^{+}$and $\sigma^{-}$[9, 11, 13]. Physically, this corresponds to factorizing out of $P(\underline{\sigma}, t)$ the quantity $P_{0}^{1 / 2}(\underline{\sigma})$, where $P_{0}(\underline{\sigma})$ is the stationary distribution in the case of simple hopping. The coagulation part reads

$$
\begin{aligned}
H_{C}=-\frac{1}{4} \sum_{i=1}^{L-1}\{ & \left(q^{-1} \sigma_{i}^{-}+\Delta q \sigma_{i}^{+}\right)\left(1+\sigma_{i+1}^{z}\right)+\left(1+\sigma_{i}^{z}\right)\left(q \sigma_{i+1}^{-}+\Delta q^{-1} \sigma_{i+1}^{+}\right) \\
& -\left(q+q^{-1}\right)(1-\Delta) \sigma_{i}^{z} \sigma_{i+1}^{z}-\left(q+q^{-1}\right)\left(\sigma_{i}^{z}+\sigma_{i+1}^{z}\right) \\
& \left.+\Delta\left(q-q^{-1}\right)\left(\sigma_{i}^{z}-\sigma_{i+1}^{z}\right)-\left(q+q^{-1}\right)(1+\Delta)\right\} .
\end{aligned}
$$

It is more complicated than $H_{D}$ because of the single spin terms $\sigma_{i}^{+}, \sigma_{i}^{-}, \sigma_{i}^{z}$ which appear in it. One should note, however, that the contribution $\left(\sigma_{i}^{z}-\sigma_{i+1}^{z}\right)$ is effectively only a boundary 
term. For $q=1$ it vanishes altogether and $H$ reduces to the expression found previously [9, 21]. In that case, $H$ was brought into a simpler form by two local transformations in spin space: A hyperbolic rotation around the $z$-axis with generator

$$
V_{1}=\prod_{n} e^{\lambda \sigma_{n}^{z}} ; \quad e^{-2 \lambda}=\sqrt{\Delta}
$$

which rescales the $\sigma^{ \pm}$operators, and a real rotation around the $y$-axis with generator

$$
V_{2}=\prod_{n} e^{-i \frac{\Theta}{2} \sigma_{n}^{y}} ; \quad \cos (\Theta)=\frac{1}{\sqrt{1+\Delta}}
$$

which forms linear contributions of $\sigma^{x}$ and $\sigma^{z}$.

It turns out that the same transformation can be used also for $q \neq 1$. The result for the new operator $H^{\prime}=V_{2} V_{1} H V_{1}^{-1} V_{2}^{-1}$ then is

$$
\begin{aligned}
H^{\prime}=-\frac{D}{2} \sum_{i=1}^{L-1} & \left\{\gamma \sigma_{i}^{x} \sigma_{i+1}^{x}+\gamma^{-1} \sigma_{i}^{y} \sigma_{i+1}^{y}+i \frac{1-q^{2}}{1+q^{2}}\left(\sigma_{i}^{x} \sigma_{i+1}^{y}-\sigma_{i}^{y} \sigma_{i+1}^{x}\right)\right. \\
& -\frac{2}{q+q^{-1}}\left(q^{-1} \sigma_{i}^{z}+q \sigma_{i+1}^{z}\right)-\left(\gamma+\gamma^{-1}\right) \\
& \left.-\frac{1-q^{2}}{1+q^{2}} \sqrt{\gamma-\gamma^{-1}}\left[\sqrt{\gamma}\left(\sigma_{i}^{x}-\sigma_{i+1}^{x}\right)+i \frac{1}{\sqrt{\gamma}}\left(\sigma_{i}^{y}-\sigma_{i+1}^{y}\right)\right]\right\}
\end{aligned}
$$

where $\gamma=\sqrt{1+\Delta}$. and $D=\frac{1}{2}\left(q+q^{-1}\right) \gamma$.

This operator has two non-hermitean parts: the third term, which is a kind of chiral contribution from the bulk, and the last term which again acts only at the two boundaries. Both vanish in the symmetric case $(q=1)$ and $H^{\prime}$ then reduces to the dual of the Glauber model operator.

The bulk terms in $H^{\prime}$ are all quadratic in fermion operators after a Jordan-Wigner transformation. There are, however, also boundary terms in $\sigma^{x}, \sigma^{y}$ and $\sigma^{z}$. The last one is unproblematic, but $\sigma^{x}$ and $\sigma^{y}$ are linear in the fermions. There is, however, a well-known way around this difficulty: One extends the chain by one site at each end and uses the additional spin operators, which are constants of the motion, to make boundary terms quadratic. Thus the operator $H^{\prime}$ is solvable via fermions.

Actually, one can bring it into a slightly simpler form by performing a rotation by $180^{\circ}$

$$
V_{3}=\prod_{n} e^{i \frac{\pi}{2} \sigma_{n}^{y}}
$$

and a further transformation with the generator

$$
V_{4}=\prod_{n} e^{\rho \sigma_{n}^{z}} ; \quad e^{-2 \rho}=\sqrt{\frac{\gamma-1}{\gamma+1}}
$$

to obtain

$$
\begin{aligned}
H^{\prime \prime}=-\frac{1}{2} \gamma \sum_{i=1}^{L-1}\{ & \left(\eta+\eta^{-1}\right) \sigma_{i}^{x} \sigma_{i+1}^{x}+i\left(q \sigma_{i}^{x} \sigma_{i+1}^{y}+q^{-1} \sigma_{i}^{y} \sigma_{i+1}^{x}\right) \\
& \left.+\left(q^{-1} \sigma_{i}^{z}+q \sigma_{i+1}^{z}\right)-\frac{1}{2}\left(q-q^{-1}\right)\left(\gamma-\gamma^{-1}\right)\left(\sigma_{i}^{x}-\sigma_{i+1}^{x}\right)-\left(\eta+\eta^{-1}\right)\right\}
\end{aligned}
$$


where $\left(\eta+\eta^{-1}\right)=\frac{1}{2}\left(q+q^{-1}\right)\left(\gamma+\gamma^{-1}\right)$.

There is no $\sigma^{y}$ boundary term in this expression so that the only non-hermitean part is the chiral bulk term. This operator will be diagonalized in the next section.

We mention that $H^{\prime}$ and $H^{\prime \prime}$ have a simple symmetry, namely they are invariant under the tranformation $q \leftrightarrow 1 / q$ combined with spatial reflection $i \rightarrow L-i+1$.

\section{Analysis of the spectrum}

In order to diagonalize the Hamiltonian (3.9) in terms of fermions, we write $H^{\prime \prime}=H_{0}^{\prime \prime}+H_{1}^{\prime \prime}$ where

$$
H_{1}^{\prime \prime}=\frac{\kappa}{2}\left(\sigma_{1}^{x}-\sigma_{L}^{x}\right)
$$

is the linear boundary term and

$$
\kappa=\frac{\gamma}{2}\left(q-q^{-1}\right)\left(\gamma-\gamma^{-1}\right)
$$

As outlined in the last section, one appends one site at each end of the chain and extends $H_{1}^{\prime \prime}$ by two Pauli matrices $\sigma_{0}^{x}$ and $\sigma_{L+1}^{x}$. Then

$$
\tilde{H}_{1}^{\prime \prime}=\frac{\kappa}{2}\left(\sigma_{0}^{x} \sigma_{1}^{x}-\sigma_{L}^{x} \sigma_{L+1}^{x}\right)
$$

is bilinear in fermions so that standard diagonalization methods [35] can be applied]. The eigenvectors of the extended Hamiltonian $\tilde{H}^{\prime \prime}=H_{0}^{\prime \prime}+\tilde{H}_{1}^{\prime \prime}$ include those of the original chain $H^{\prime \prime}$ as follows: Since the appended matrices are constants of the motion, the spectrum of $\tilde{H}^{\prime \prime}$ decomposes into four sectors $(++,+-,-+,--)$ corresponding to the eigenvalues of $\sigma_{0}^{x}$ and $\sigma_{L+1}^{x}$. This implies that all eigenvectors of $\tilde{H}^{\prime \prime}$ have the form

$$
|\tilde{\psi}\rangle=\left(\begin{array}{c}
1 \\
\pm 1
\end{array}\right) \otimes|\psi\rangle \otimes\left(\begin{array}{c}
1 \\
\pm 1
\end{array}\right)
$$

where $|\psi\rangle$ is a vector with $2^{L}$ components. Since in each sector the appended matrices can be replaced by their eigenvalues, the spectrum of the original chain appears in the $(++)$ sector and the vectors $|\psi\rangle$ are just the eigenvectors of $H^{\prime \prime}$. Therefore we can determine the eigenvectors of the original problem by projection onto the $(++)$ sector. One should mention that a very similar situation occurs in calculations using the corner transfer matrix of the two-dimensional Ising model [36, 37]. In that case the sectors arise from the fixed inner and outer spins in the corner geometry.

Defining anticommuting Clifford operators $\tau_{j}^{x, y}=\left(\prod_{i=0}^{j-1} \sigma_{i}^{z}\right) \sigma_{j}^{x, y}$ one can rewrite $\tilde{H}^{\prime \prime}$ as a bilinear expression in $\tau_{j}^{x}$ and $\tau_{j}^{y}$. Then a second linear transformation $a_{k}^{ \pm}=\frac{1}{2} \sum_{j=1}^{L}\left(\phi_{k, j}^{x} \tau_{j}^{x} \pm\right.$ $\left.i \phi_{k, j}^{y} \tau_{j}^{y}\right)$ yields

$$
\tilde{H}^{\prime \prime}=\left(\sum_{k} \lambda_{k} a_{k}^{+} a_{k}^{-}\right)-\kappa
$$

\footnotetext{
${ }^{3}$ In the same way one could also add $\sigma^{y}$-matrices or any linear combination of $\sigma^{x}$ and $\sigma^{y}$.
} 
which is a diagonal expression in fermionic creation and annihilation operators $a_{k}^{+}$and $a_{k}^{-}$. The one-particle energies $\lambda_{k}$ and the eigenvectors $\vec{\phi}_{k}^{ \pm}=\left(\phi_{k, 1}^{x}, \pm i \phi_{k, 1}^{y}, \ldots, \phi_{k, L}^{x}, \pm i \phi_{k, L}^{y}\right)$ can be derived from the eigenvalue problem $M \vec{\phi}_{k}^{ \pm}=\mp \lambda_{k} \vec{\phi}_{k}^{ \pm}$with

$$
M=i \gamma\left(\begin{array}{cccccccc}
0 & D & & & & & \\
-D^{T} & B & A & & & & \\
& -A^{T} & B+C & A & & & \\
& & \cdots & \cdots & \ldots & & \\
& & & -A^{T} & B+C & A & \\
& & & & -A^{T} & C & -D \\
& & & & & D^{T} & 0
\end{array}\right)
$$

where $A, B, C$ and $D$ denote the $2 \times 2$ block matrices

$$
A=\left(\begin{array}{cc}
-i / q & 0 \\
\eta+\eta^{-1} & i q
\end{array}\right), \quad B=\left(\begin{array}{cc}
0 & q^{-1} \\
-q^{-1} & 0
\end{array}\right), \quad C=\left(\begin{array}{cc}
0 & q \\
-q & 0
\end{array}\right), \quad D=\left(\begin{array}{cc}
0 & 0 \\
\kappa / \gamma & 0
\end{array}\right) .
$$

The eigenvalues of $M$ are real and occur in pairs with different signs. It should be emphasized at this point that although $a_{k}^{+}$and $a_{k}^{-}$obey the usual fermionic commutation relations $\left\{a_{k}^{+}, a_{l}^{-}\right\}=\delta_{k l}$, we have $a_{k}^{+} \neq\left(a_{k}^{-}\right)^{\dagger}$ because of the nonhermiticity of $\tilde{H}^{\prime \prime}$.

Assuming that the wave function $\phi_{k, j}^{ \pm}$consists of exponential contributions $e^{-\zeta j}$, the bulk equations (4.6) yield the dispersion relation

$$
\lambda=\gamma\left(\eta+\eta^{-1}-q e^{-\zeta}-q^{-1} e^{\zeta}\right) .
$$

Including the boundary conditions which determine $\zeta$, we obtain three types of fermionic one-particle states:

- One trivial zero mode $\left(\lambda_{0}=0\right)$ which is localized at the ends of the extended chain. This zero mode represents conserved quantities $\sigma_{0}^{x}$ and $\sigma_{L+1}^{x}$ and causes two-fold degeneracies of all levels.

- Two exponential modes with excitation energies $\lambda_{I}=\lambda_{I I}=\kappa$. Their wave functions $\phi_{I}^{ \pm}$and $\phi_{I I}^{ \pm}$decay exponentially with three different length scales corresponding to the values $\ln (\gamma), \ln \left(q^{2} \gamma\right)$ and $\ln \left(q^{2} \gamma^{-1}\right)$ for $\zeta$. These modes reflect the influence of the boundary term $H_{1}^{\prime \prime}$.

- $L-1$ oscillatory modes with $\lambda_{k}=\gamma\left(\eta+\eta^{-1}\right)-2 \gamma \cos \frac{\pi k}{L}$ where $k=1, \ldots, L-1$. The corresponding wave functions show damped oscillations of the form $\phi_{k, j} \sim q^{ \pm j} e^{ \pm i \pi k / L}$. This implies the presence of a fourth length scale with $\zeta=\ln q$. The damping is a consequence of the nonhermiticity of the Hamiltonian for $q \neq 1$.

One can show that the lowest level of $\tilde{H}^{\prime \prime}$ is a two-fold degenerate state in the sectors $(+-)$ and $(-+)$ with a negative eigenvalue $\Lambda=-\kappa$. The lowest level in the $(++)$ sector with eigenvalue zero is already an excited state. The total number of excited modes in this sector has to be odd and one can distinguish: 
- States with an even number of excited oscillatory modes. The levels are two-fold degenerate because either mode $I$ or mode $I I$ can be occupied. This class contains the ground state with $\Lambda=0$ and the lowest gap is

$$
\Delta \Lambda=\gamma\left(\eta+\frac{1}{\eta}-2\right) .
$$

- States with an odd number of excited oscillatory modes. Here the modes $I$ and $I I$ are either both occupied, which gives a contribution $(+\kappa)$ to $\Lambda$, or both empty, which gives $(-\kappa)$, i.e. we have two bands of excitations which are shifted symmetrically by $\pm \kappa$. Their lowest gaps are

$$
\Delta \Lambda(\kappa)=\gamma\left(\gamma q+\frac{1}{\gamma q}-2\right), \quad \Delta \Lambda(-\kappa)=\gamma\left(\frac{\gamma}{q}+\frac{q}{\gamma}-2\right) .
$$

The nature of these three branches (singlets and doublets) suggests a symmetry in the problem. This is indeed the case: From the operators for the two exponential modes one can construct the quantities

$$
\begin{array}{r}
\sigma=1-\left(a_{I}^{+} a_{I}^{-}+a_{I I}^{+} a_{I I}^{-}\right)+\left(a_{I}^{+} a_{I I}^{-}+a_{I I}^{+} a_{I}^{-}\right) \\
\tau=1-\frac{3}{2}\left(a_{I}^{+} a_{I}^{-}-a_{I I}^{+} a_{I I}^{-}\right)^{2}-\frac{\sqrt{3}}{2}\left(a_{I}^{+} a_{I I}^{-}-a_{I I}^{+} a_{I}^{-}\right) .
\end{array}
$$

They satisfy the relations

$$
\sigma^{2}=1, \quad \tau^{3}=1, \quad \sigma \tau \sigma=\tau^{2}
$$

which characterize the generators of the permutation group $S_{3}$ and commute with both $\tilde{H}^{\prime \prime}$ and $H^{\prime \prime}$. Thus the levels can be chosen as irreducible representations of $S_{3}$ which, according to group theory [38], consist of two types of singlets and one doublet. The operator $\sigma$ has a simple meaning, it interchanges the two degenerate fermion modes.

The smallest gap is obtained from one of the expressions (4.9) which differ by a reflection $q \rightarrow q^{-1}$. It vanishes if $\gamma=q(q>1)$ or $\gamma=q^{-1}(q<1)$. One band of excitations is then massless and algebraic long-time behaviour is expected. At this point a transition takes place between a low-density phase (for $\gamma<q$ ) and a high-density phase (for $\gamma>q$ ). This will be seen in section 7 from the steady-state properties of the system. One should note, however, that the situation is different from a Pokrovskii-Talapov type of transition [39] since here the spectrum has a gap on both sides $(\gamma \lesseqgtr q)$ and always varies quadratically with the momentum $k$.

\section{Hole formalism}

Although the diagonalization in terms of fermions solves the model in principle, another complementary approach is simpler for practical purposes and gives additional insight. In this approach the states of the system are described in terms of strings of empty sites (holes) instead of spin configurations [17]. In this way one can distinguish different sectors characterized by the number of holes. The equations for the probabilities couple a sector 
only to the one with lower order. For the one-hole sector of the coagulation model (2.7) with free ends they were already derived in Ref. [24]. Denoting by $\Omega(x, y, t)$ the probability to find the sites $x+1, \ldots, y$ empty and using the abbreviations

$$
\begin{aligned}
\alpha_{L, R} & =a_{L, R}+d_{L, R}-b_{L, R}-c \\
\gamma_{L, R} & =a_{L}+a_{R}+d_{L, R} \\
\delta & =b_{L}+b_{R}+c
\end{aligned}
$$

they read

- for holes which do not touch the boundaries $(0<x<y<L)$ :

$$
\begin{aligned}
\frac{d}{d t} \Omega(x, y, t)= & \alpha_{R} \Omega(x-1, y, t)+a_{L} \Omega(x+1, y, t) \\
& +a_{R} \Omega(x, y-1, t)+\alpha_{L} \Omega(x, y+1, t) \\
& -\left(\gamma_{L}+\gamma_{R}+(y-x-1) \delta\right) \Omega(x, y, t)
\end{aligned}
$$

- for holes touching the left boundary $(0=x<y<L)$ :

$$
\begin{aligned}
\frac{d}{d t} \Omega(0, y, t)= & a_{R} \Omega(0, y-1, t)+\alpha_{L} \Omega(0, y+1, t) \\
& -\left(\gamma_{L}+(y-1) \delta\right) \Omega(0, y, t)
\end{aligned}
$$

- for holes touching the right boundary $(0<x<y=L)$ :

$$
\begin{aligned}
\frac{d}{d t} \Omega(x, L, t)= & \alpha_{R} \Omega(x-1, L, t)+a_{L} \Omega(x+1, L, t) \\
& -\left(\gamma_{R}+(L-x-1) \delta\right) \Omega(x, L, t)
\end{aligned}
$$

- for the hole extending over the whole chain $(x=0, y=L)$ :

$$
\frac{d}{d t} \Omega(0, L, t)=-(L-1) \delta \Omega(0, L, t)
$$

where we formally define $\Omega(x, x, t)=1$ according to [24]. This is the coupling to the lower sector (no holes), which here leads to an inhomogeneous system. The integrable case discussed in the preceeding sections corresponds to

$$
\alpha_{L, R}=\gamma^{2} q^{ \pm 1}, \quad a_{L, R}=q^{ \pm 1}, \quad \gamma_{L, R}=q^{\mp 1}+\gamma^{2} q^{ \pm 1}, \quad \delta=0 .
$$

Introducing rescaled probabilities $\tilde{\Omega}(x, y, t)$ via

$$
\tilde{\Omega}(x, y, t)=\gamma^{y-x} q^{y+x} \Omega(x, y, t)
$$

and separating the time dependence $\exp (-\Lambda t)$, Eqs. (5.2)-(5.5) reduce to:

$$
\begin{aligned}
\left(\gamma\left(q+q^{-1}\right)\left(\gamma+\gamma^{-1}\right)-\Lambda\right) \tilde{\Omega}(x, y)= & \gamma(\tilde{\Omega}(x-1, y)+\tilde{\Omega}(x+1, y) \\
& +\tilde{\Omega}(x, y-1)+\tilde{\Omega}(x, y+1)) \\
\left(q^{-1}+\gamma^{2} q-\Lambda\right) \tilde{\Omega}(0, y)= & \gamma(\tilde{\Omega}(0, y-1)+\tilde{\Omega}(0, y+1)) \\
\left(q+\gamma^{2} q^{-1}-\Lambda\right) \tilde{\Omega}(x, L)= & \gamma(\tilde{\Omega}(x+1, L)+\tilde{\Omega}(x-1, L)) \\
\Lambda \tilde{\Omega}(0, L)= & 0
\end{aligned}
$$


with the inhomogeneous boundary condition $\tilde{\Omega}(x, x)=q^{2 x}$. These equations can be solved using similar techniques as in Ref. 21] which rely mainly on the invariance of the bulk equation (5.8) under reflections $x \leftrightarrow y$ and $x \leftrightarrow L-y$. A trivial inhomogeneous solution is the empty lattice, for which $\tilde{\Omega}(x, y)=\gamma^{y-x} q^{y+x}$. The homogeneous set of solutions satisfies the condition $\tilde{\Omega}(x, x)=0$ and reads

$$
\begin{aligned}
\Phi_{0}(x, y) & =h(L-x) g(y)-h(L-y) g(x) \\
\Lambda_{0} & =0 \\
\Phi_{k}^{(L)}(x, y) & =\sin \frac{\pi k x}{L} h(L-y)-\sin \frac{\pi k y}{L} h(L-x) \\
\Lambda_{k}^{(L)} & =\gamma\left(\gamma q+\gamma^{-1} q^{-1}-2 \cos \frac{\pi k}{L}\right) \\
\Phi_{k}^{(R)}(x, y) & =\sin \frac{\pi k x}{L} g(y)-\sin \frac{\pi k y}{L} g(x) \\
\Lambda_{k}^{(R)} & =\gamma\left(\gamma q^{-1}+\gamma^{-1} q-2 \cos \frac{\pi k}{L}\right) \\
\Phi_{k, l}(x, y) & =\sin \frac{\pi k x}{L} \sin \frac{\pi l y}{L}-\sin \frac{\pi k y}{L} \sin \frac{\pi l x}{L} \\
\Lambda_{k, l} & =\gamma\left(\left(q+q^{-1}\right)\left(\gamma+\gamma^{-1}\right)-2\left(\cos \frac{\pi k}{L}+\cos \frac{\pi l}{L}\right)\right)
\end{aligned}
$$

where $1 \leq k<l \leq L-1$ and the functions $g$ and $h$ are

$$
g(x)=\frac{(\gamma q)^{x}-(\gamma q)^{-x}}{(\gamma q)^{L}-(\gamma q)^{-L}}, \quad h(x)=\frac{(\gamma / q)^{x}-(\gamma / q)^{-x}}{(\gamma / q)^{L}-(\gamma / q)^{-L}}
$$

We can now compare the eigenvalues $\Lambda_{0}, \Lambda_{k}^{L, R}$ and $\Lambda_{k, l}$ with the spectrum computed in the last section. As in the symmetric case [21], $\Lambda_{0}$ gives a two-fold degenerate ground state while $\Lambda_{k}^{L, R}$ and $\Lambda_{k, l}$ are seen to be one- and two-particle excitations. However, in contrast to the symmetric case we have $\Lambda_{k}^{L} \neq \Lambda_{k}^{R}$ since these levels are shifted by $\pm \kappa$. Therefore the boundary effects of $\tilde{H}_{2}^{\prime \prime}$ do not change the selection rules, they only modify the mass gaps of the one-particle modes.

\section{Probabilities for $\mathrm{n}$ holes}

For a complete treatment of the system one has to study configurations with an arbitrary number of holes. This was already discussed in Ref. 24] and we will now carry out such a study. The probability to find the strings of sites $\left(x_{1}+1, \cdots, y_{1}\right),\left(x_{2}+1, \cdots, y_{2}\right), \cdots,\left(x_{n}+\right.$ $\left.1, \cdots, y_{n}\right)$ with $0 \leq x_{1}<\cdots<y_{n} \leq L$ empty at time $t$ is denoted by $\Omega_{n}\left(x_{1}, y_{1}, \cdots, x_{n}, y_{n}, t\right)$ and will also be called $n$-hole function. If no hole extends up to the boundaries, the time evolution of $\Omega_{n}$ takes the form :

$$
\frac{d}{d t} \Omega_{n}\left(x_{1}, y_{1}, \cdots, x_{n}, y_{n}, t\right)=\sum_{i=1}^{n}\left[-\left(\gamma_{L}+\gamma_{R}+\left(y_{i}-x_{i}-1\right) \delta\right) \Omega_{n}\left(x_{1}, y_{1}, \cdots, x_{n}, y_{n}, t\right)\right.
$$




$$
\begin{aligned}
& +\alpha_{R} \Omega_{n}\left(\cdots, x_{i}-1, y_{i}, \cdots, t\right)+a_{L} \Omega_{n}\left(\cdots, x_{i}+1, y_{i}, \cdots, t\right) \\
& \left.+a_{R} \Omega_{n}\left(\cdots, y_{i}-1, x_{i+1}, \cdots, t\right)+\alpha_{L} \Omega_{n}\left(\cdots, y_{i}+1, x_{i+1}, \cdots, t\right)\right]
\end{aligned}
$$

with the parameters defined in (5.1). The $i^{\text {th }}$ term in the sum on the right hand side can be identified as time evolution of a one-hole function (5.2). In the following we choose the reaction rates according to $(2.8)$ and rescale the $\Omega_{n}$ as in the previous section :

$$
\tilde{\Omega}_{n}\left(x_{1}, y_{1}, \cdots, x_{n}, y_{n}, t\right)=\gamma^{\sum_{i=1}^{n}\left(y_{i}-x_{i}\right)} q^{\sum_{i=1}^{n}\left(x_{i}+y_{i}\right)} \Omega_{n}\left(x_{1}, y_{1}, \cdots, x_{n}, y_{n}, t\right)
$$

Then the rescaled time evolution equation turns out to be invariant under an arbitrary permutation of the variables $\left(x_{1}, \cdots, y_{n}\right)$. This symmetry can be taken into account by introducing new coordinates $\left(z_{1}, z_{2}, \cdots, z_{2 n-1}, z_{2 n}\right):=\left(x_{1}, y_{1}, \cdots, x_{n}, y_{n}\right)$. The complete system of equations then reads:

- if no holes touch the boundaries $\left(0<z_{1}, z_{2 n}<L\right)$ :

$$
\begin{aligned}
\frac{d}{d t} \tilde{\Omega}_{n}\left(z_{1}, \cdots, z_{2 n}, t\right)= & -n \gamma\left(\gamma+\gamma^{-1}\right)\left(q+q^{-1}\right) \tilde{\Omega}_{n}\left(z_{1}, \cdots, z_{2 n}, t\right) \\
& +\gamma \sum_{i=1}^{2 n}\left[\tilde{\Omega}_{n}\left(\cdots, z_{i}-1, z_{i+1}, \cdots, t\right)+\tilde{\Omega}_{n}\left(\cdots, z_{i}+1, z_{i+1}, \cdots, t\right)\right]
\end{aligned}
$$

- if the leftmost hole touches the boundary $\left(0=z_{1}, z_{2 n}<L\right)$ :

$$
\begin{gathered}
\frac{d}{d t} \tilde{\Omega}_{n}\left(0, z_{2}, \cdots, z_{2 n}, t\right)=-\left((n-1) \gamma\left(\gamma+\gamma^{-1}\right)\left(q+q^{-1}\right)+\gamma^{2} q+q^{-1}\right) \tilde{\Omega}_{n}\left(z_{1}, \cdots, z_{2 n}, t\right) \\
+\gamma \sum_{i=2}^{2 n}\left[\tilde{\Omega}_{n}\left(\cdots, z_{i}-1, z_{i+1}, \cdots, t\right)+\tilde{\Omega}_{n}\left(\cdots, z_{i}+1, z_{i+1}, \cdots, t\right)\right]
\end{gathered}
$$

- if the rightmost hole touches the boundary $\left(0<z_{1}, z_{2 n}=L\right)$ :

$$
\begin{gathered}
\frac{d}{d t} \tilde{\Omega}_{n}\left(z_{1}, \cdots, z_{2 n-1}, L, t\right)=-\left((n-1) \gamma\left(\gamma+\gamma^{-1}\right)\left(q+q^{-1}\right)+\gamma^{2} q+q^{-1}\right) \tilde{\Omega}_{n}\left(z_{1}, \cdots, z_{2 n}, t\right) \\
+\gamma \sum_{i=1}^{2 n-1}\left[\tilde{\Omega}_{n}\left(\cdots, z_{i}-1, z_{i+1}, \cdots, t\right)+\tilde{\Omega}_{n}\left(\cdots, z_{i}+1, z_{i+1}, \cdots, t\right)\right]
\end{gathered}
$$

- if the leftmost and the rightmost holes touch the boundaries $\left(0=z_{1}, z_{2 n}=L\right)$ :

$$
\begin{array}{r}
\frac{d}{d t} \tilde{\Omega}_{n}\left(0, z_{2}, \cdots, z_{2 n-1}, L, t\right)=-(n-1) \gamma\left(\gamma+\gamma^{-1}\right)\left(q+q^{-1}\right) \tilde{\Omega}_{n}\left(z_{1}, \cdots, z_{2 n}, t\right) \\
+\gamma \sum_{i=2}^{2 n-1}\left[\tilde{\Omega}_{n}\left(\cdots, z_{i}-1, z_{i+1}, \cdots, t\right)+\tilde{\Omega}_{n}\left(\cdots, z_{i}+1, z_{i+1}, \cdots, t\right)\right]
\end{array}
$$

However, this set of linear equations for the $n$-hole functions is not closed since on the right hand side terms with $z_{i}=z_{i+1}$ appear. These terms have to be identified with $(n-1)$-hole functions according to

$$
\tilde{\Omega}_{n}\left(z_{1}, \cdots, z_{2 n}\right)=q^{2 z_{i}} \tilde{\Omega}_{n-1}\left(z_{1}, \cdots, z_{i-1}, z_{i+2}, \cdots, z_{2 n}\right) \text { if } z_{i}=z_{i+1}
$$

and therefore cause a coupling to the lower sectors as mentioned before. This coupling constitutes the main difficulty in solving the equations. A similar situation occurs for the 
spin correlation functions of the Glauber model, and in that case a method to solve the problem was developed by Bedeaux, Schuler and Oppenheim [27. It can be applied in the present case, too. For that purpose we introduce new cumulant-like functions $C_{n}$ with the property that

(1) $C_{n}$ obeys the differential equations 6.3$)-(6.6)$

(2) $C_{n}\left(z_{1}, \cdots, z_{2 n}, t\right)=0$ if $z_{i}=z_{i+1}$

Following Ref. [27], these functions are defined by

$$
\begin{aligned}
C_{n}\left(z_{1}, \cdots, z_{2 n}, t\right)= & \sum_{\xi}(-1)^{|P|}(-1)^{(k-1)}(k-1) ! \tilde{\Omega}_{n_{1}}\left(z_{1}^{\xi}, \cdots, z_{2 n_{1}}^{\xi}, t\right) \\
& \quad \times \tilde{\Omega}_{n_{2}}\left(z_{2 n_{1}+1}^{\xi}, \cdots, z_{2 n_{1}+2 n_{2}}^{\xi}, t\right) \cdots \tilde{\Omega}_{n_{k}}\left(z_{2 n-2 n_{k}+1}^{\xi}, \cdots, z_{2 n}^{\xi}, t\right) .
\end{aligned}
$$

The sum on the right hand side of (6.8) runs over partitions $\xi$ of the numbers $z_{1}, \cdots, z_{2 n}$ into $k$ subsets $\left(z_{1}^{\xi}, \cdots, z_{2 n_{1}}^{\xi}\right),\left(z_{2 n_{1}+1}^{\xi}, \cdots, z_{2 n_{1}+2 n_{2}}^{\xi}\right), \cdots,\left(z_{2 n-2 n_{k}+1}^{\xi}, \cdots, z_{2 n}^{\xi}\right)$, with sizes $n_{j}$ where $j=1, \cdots, k$. Within these subsets the $z_{i}^{\xi}$ are ordered according to their magnitude. $P$ is the permutation which transforms $\left(z_{1}, \cdots, z_{2 n}\right)$ into $\left(z_{1}^{\xi}, \cdots, z_{2 n}^{\xi}\right)$ and $|P|$ denotes its (uniquely defined) sign. The first two functions $C_{1}$ and $C_{2}$ read explicitly:

$$
\begin{aligned}
C_{1}\left(z_{1}, z_{2}, t\right)= & \tilde{\Omega}_{1}\left(z_{1}, z_{2}, t\right) \\
C_{2}\left(z_{1}, z_{2}, z_{3}, z_{4}, t\right)= & \tilde{\Omega}_{2}\left(z_{1}, z_{2}, z_{3}, z_{4}, t\right)-\tilde{\Omega}_{1}\left(z_{1}, z_{2}, t\right) \tilde{\Omega}_{1}\left(z_{3}, z_{4}, t\right) \\
& -\tilde{\Omega}_{1}\left(z_{1}, z_{4}, t\right) \tilde{\Omega}_{1}\left(z_{2}, z_{3}, t\right)+\tilde{\Omega}_{1}\left(z_{1}, z_{3}, t\right) \tilde{\Omega}_{1}\left(z_{2}, z_{4}, t\right)
\end{aligned}
$$

The proof that the $C_{n}$ have the property (2) by which the sectors are decoupled, is completely analogous to the one given in [27]. Property (1) holds since each term on the right hand side of equation (6.8) solves the differential equation (6.3)-(6.6).

However, this is only true if the conditions $\delta=0$ and $\alpha_{R} a_{L}=\alpha_{L} a_{R}$ are satisfied so that the rescaled equations (6.3)-(6.6) are symmetric in the starting points $x$ and end points $y$ of the holes. In the general case, i.e. for arbitrary values of the parameters $\delta, \alpha_{L}, \alpha_{R}, a_{L}$ and $a_{R}$, this symmetry is lost and arbitrary products of $\tilde{\Omega}$-functions are no longer solutions of the generalisation of (6.3)-(6.6). Then the given construction of the $C$-functions fails. It may not be so surprising that the method of Bedeaux et.al., which was developed for the Glauber model, works also here only in the free fermion case. To get an idea whether the general case is integrable or not, one can apply Reshetikhin's criterion for integrability [28]:

$$
\left[H_{n}+H_{n+1},\left[H_{n}, H_{n+1}\right]\right]=X_{n}-X_{n+1}
$$

to the Hamiltonian (2.7), where $X_{n}$ is an arbitrary $4 \times 4$ matrix. We found that it leads to the same restriction on the reaction rates, namely $\delta=0$ and $\alpha_{R} a_{L}=\alpha_{L} a_{R}$. It is known, however, that this criterion is not a necessary condition.

Turning to the solution of the equations, one first observes that they are inhomogeneous only for $n=1$. A particular solution $C^{P}$ is again the empty lattice:

$$
C_{1}^{P}\left(z_{1}, z_{2}\right)=\gamma^{\left(z_{2}-z_{1}\right)} q^{\left(z_{2}+z_{1}\right)}, \quad C_{n}^{P}=0 \text { for } n \geq 2 .
$$


For the solutions of the homogeneous system we write

$$
C_{n}^{H}\left(z_{1}, \cdots, z_{2 n}, t\right)=e^{-\Lambda t} \Phi^{(n)}\left(z_{1}, \cdots, z_{2 n}\right)
$$

and are left with an eigenvalue problem as in the previous section. As there, one finds four types of solutions (c.f. Eqs. (5.12)-(5.15)):

$$
\begin{array}{r}
\Phi_{k_{1}, k_{2}, \cdots, k_{2 n}}^{(n)}=\sum_{\tau \epsilon S_{2 n}}(-1)^{|\tau|} \prod_{i=1}^{2 n} \sin \frac{\pi k_{i}}{L} z_{\tau(i)} \\
\Lambda=n \gamma\left(\gamma+\gamma^{-1}\right)\left(q+q^{-1}\right)-2 \gamma \sum_{i=1}^{2 n} \cos \frac{\pi k_{i}}{L} \\
\Phi_{k_{2}, k_{3}, \cdots, k_{2 n}}^{(n, L)}=\sum_{\tau \epsilon S_{2 n}}(-1)^{|\tau|}\left(h\left(L-z_{\tau(1)}\right) \prod_{i=2}^{2 n} \sin \frac{\pi k_{i}}{L} z_{\tau(i)}\right) \\
\Lambda=\left((n-1) \gamma\left(\gamma+\gamma^{-1}\right)\left(q+q^{-1}\right)+\gamma^{2} q+q^{-1}\right)-2 \gamma \sum_{i=2}^{2 n} \cos \frac{\pi k_{i}}{L} \\
\Phi_{k_{1}, k_{2}, \cdots, k_{2 n-1}}^{(n, R)} \sum_{\tau \epsilon S_{2 n}}(-1)^{|\tau|}\left(\prod_{i=1}^{2 n-1} \sin \frac{\pi k_{i}}{L} z_{\tau(i)} g\left(z_{\tau(2 n)}\right)\right) \\
\Lambda=\left((n-1) \gamma\left(\gamma+\gamma^{-1}\right)\left(q+q^{-1}\right)+\gamma^{2} q+q^{-1}\right)-2 \gamma \sum_{i=1}^{2 n-1} \cos \frac{\pi k_{i}}{L} \\
\Phi_{k_{2}, k_{3}, \cdots, k_{2 n-1}}^{(n, L, R)}=\sum_{\tau \in S_{2 n}}(-1)^{|\tau|}\left(h\left(L-z_{\tau(1)}\right) \prod_{i=1}^{2 n-1} \sin \frac{\pi k_{i}}{L} z_{\tau(i)} g\left(z_{\tau(2 n)}\right)\right) \\
\Lambda=(n-1) \gamma\left(\gamma+\gamma^{-1}\right)\left(q+q^{-1}\right)-2 \gamma \sum_{i=2}^{2 n-1} \cos \frac{\pi k_{i}}{L}
\end{array}
$$

In these equations the $k_{i}$ are integers with $1 \leq k_{1}<\cdots<k_{2 n} \leq L-1$. The sum runs over all elements of the permutation group $S_{2 n}$. To understand these solutions we remark that each term in the sum of (6.146.17) solves equations (6.3-6.6) up to boundary conditions. These are satified by taking the totaly antisymmetric combination. In the case $n=1$ one recovers the results of section 5 .

Comparing the eigenvalues $\Lambda$ with the spectrum given in section 4 we find that they correspond to the excitation of $2 n, 2 n-1$ and $2(n-1)$ fermions for the cases (6.14), (6.15)(6.16) and (6.17), respectively. Furthermore, one has as many solutions as $n$-hole functions. Therefore the system of eigenfunctions found above is complete and the problem is thereby fully solved.

\section{$7 \quad$ Steady state properties}

The simplest application of the previous results is the investigation of stationary properties.

The system has two steady states, namely a trivial one (the empty lattice) and a nontrivial 
one where particles are present. Considering the situation where at least one particle is present (i.e. $\left.\Omega_{\text {stat }}(0, L)=0\right)$ we have:

$$
\Omega_{\text {stat }}(x, y)=1-\gamma^{(L-y+x)} q^{(L-y-x)} \Phi_{0}(x, y)
$$

Inserting $\Phi_{0}$ from Eq. (5.12) then gives the following exact expression for the concentration $c(j)=1-\Omega_{\text {stat }}(j-1, j)$ at site $j$ :

$$
c(j)=\frac{1}{K}\left\{\gamma^{2 L}\left(\left(\gamma^{2}-1\right)+\left(q^{2}-1\right) \gamma^{2}(q \gamma)^{-2 j}\right)-q^{2 L}\left(\left(\gamma^{2}-1\right) q^{2-4 j}+\left(q^{2}-1\right)(q / \gamma)^{-2 j}\right)\right\}
$$

where

$$
K=\gamma^{2}\left(\gamma^{2 L}+\gamma^{-2 L}-q^{2 L}-q^{-2 L}\right)
$$

The density profile $c(j)$ is shown in Fig. 1 for 100 sites, a fixed asymmetry $q=1.2$ towards the left and various decoagulation rates $\Delta$. As can be seen, there are three types of curves: If $\Delta$ is small, particles are found only near the preferred (left) boundary, while for large $\Delta$ a plateau exists together with some additional boundary effects. At the border between these two cases, the concentration decays linearly in the bulk. Computing the leading order of $c(j)$ for $L \rightarrow \infty$ gives the following explicit forms for the profile

$$
c(j)= \begin{cases}\frac{\Delta}{1+\Delta} q^{2-4 j}+\frac{q^{2}-1}{1+\Delta}\left(\frac{q}{\gamma}\right)^{-2 j} & \text { if } \Delta<q^{2}-1 \\ \frac{\Delta}{1+\Delta}\left(q^{2-4 j}+1-\frac{j}{L}\right) & \text { if } \Delta=q^{2}-1 \\ \frac{\Delta}{1+\Delta}+\left(q^{2}-1\right)\left((q \gamma)^{-2 j}-\left(\frac{q}{\gamma}\right)^{2 L-2 j}\right) & \text { if } \Delta>q^{2}-1\end{cases}
$$

From these expressions one sees that three different inverse length scales given by $4 \ln (q)$, $2 \ln (q / \gamma)$ and $2 \ln (q \gamma)$ appear which are related to those found in the fermion eigenfunctions of section 4 . The mean concentration per site $\bar{c}$ in leading order for $L \rightarrow \infty$ is

$$
\bar{c}=\left\{\begin{array}{ccc}
\frac{A}{L} & \text { if } & \Delta<q^{2}-1 \\
\frac{1}{2} \frac{\Delta}{1+\Delta} & \text { if } & \Delta=q^{2}-1 \\
\frac{\Delta}{1+\Delta} & \text { if } & \Delta>q^{2}-1
\end{array}\right.
$$

where

$$
A=\frac{\left(1+\Delta-q^{4}\right)\left(1+\Delta-q^{-2}\right)}{\left(1+\Delta-q^{2}\right)(1+\Delta)\left(q^{2}-q^{-2}\right)} \geq 1 .
$$

Thus we have to distinguish two different phases. In the low-density phase $\Delta<q^{2}-1$ the asymmetric diffusion is strong enough to move the bulk particles to the boundaries where they can coagulate. Here one has a stationary state with a finite number of particles $A \geq 1$ and therefore the mean concentration $\bar{c}$ is of order $1 / L$. In the high-density phase $\Delta>q^{2}-1$, the bulk concentration $\frac{\Delta}{1+\Delta}$ is finite and independent of the asymmetry $q$ so that $\bar{c}$ is of order one. At the transition $\Delta=q^{2}-1$ (i.e. $q=\gamma$ or $q=\gamma^{-1}$ ), one of the lengths diverges and one has a linear decay of the concentration in the bulk. This is also the point where the gap in the spectrum vanishes (c.f. section 4).

This phase structure also shows up in the stationary two-point correlation function. 
Denoting by $n_{i}$ and $n_{j}$ the occupation numbers at the positions $i<j$, its connected part is given by

$$
\begin{aligned}
g(i, j) & =\left\langle n_{i} n_{j}\right\rangle-\left\langle n_{i}\right\rangle\left\langle n_{j}\right\rangle \\
& =\left\langle\left(1-n_{i}\right)\left(1-n_{j}\right)\right\rangle-\left\langle\left(1-n_{i}\right)\right\rangle\left\langle\left(1-n_{j}\right)\right\rangle \\
& =\Omega_{2}^{\text {stat }}(i-1, i, j-1, j)-\Omega_{1}^{\text {stat }}(i-1, i) \Omega_{1}^{\text {stat }}(j-1, j) \\
& =\gamma^{2(j-i-1)}\left(\Omega_{1}^{\text {stat }}(i-1, j) \Omega_{1}^{\text {stat }}(i, j-1)-\Omega_{1}^{\text {stat }}(i-1, j-1) \Omega_{1}^{\text {stat }}(i, j)\right)
\end{aligned}
$$

To derive the last formula one uses Eqs. (6.9), (6.10) and the f act that for stationary states all functions $C_{n}$ for $n \geq 2$ vanish because their relaxational spectrum has no zero eigenvalues. Inserting the expression for the funtion $\Omega_{1}^{s t a t}(i, j)$ one has:

$$
\begin{aligned}
g(i, j)=\gamma^{L+j-i-3} q^{L-j-i+1} & {\left[\left(h(L-i+1)-\left(\gamma q^{-1}\right) h(L-i)\right)((\gamma q) g(j-1)-g(j))\right.} \\
& \left.-((\gamma q) h(L-j+1)-h(L-j))\left(g(i-1)-\left(\gamma q^{-1}\right) g(i)\right)\right] \\
& -c(i) c(j)
\end{aligned}
$$

In the symmetric case $(q=1)$ the system has a ground state of product form. Therefore the particle concentration $\Delta /(1+\Delta)$ equals the mean field value and the connected correlation function is zero. If there is an asymmetry to the left $(q>1)$, one finds the following properties

- In the low-density phase $\Delta<q^{2}-1$ the only region where correlations are present is the left boundary. Here the correlation function is negative since the dominating coagulation process reduces the probability that particles meet each other.

- In the high-density phase $\Delta>q^{2}-1$ there are correlations at both boundaries. As in the previous case they are negative at the left boundary and vanish in the bulk. At the right boundary where the particle concentration is reduced, decoagulation is the dominating process which increases the probability of neighbouring particles.

- At the phase transition $\Delta=q^{2}-1$ there is a linear decay of the correlation function for large distances. For large $L$ and $i / L, j / L$ fixed one has

$$
g(i / L, j / L)=\left(\frac{\Delta}{1+\Delta}\right)^{2}(i / L)(1-j / L)
$$

For short distances the correlations are nontrivial only near the left boundary where they have exponential form. For large $L$ and $i, j$ fixed

$$
g(i, j)=-\left(\frac{\Delta}{1+\Delta}\right)^{2}\left(1+\gamma^{-4 i+2}\right) \gamma^{-4 j+2}
$$

In this case the remaining length scale $1 / 4 \ln (\gamma)$ enters and describes the boundary effect. 


\section{Concluding remarks}

We have studied the combined effect of a preferred direction and of open boundaries in a one-dimensional coagulation model. By formulating it as a quantum spin chain, the complete integrability was shown. The relaxation spectrum, although obtained from a non-hermitean operator, turned out to be real, with a gap determined by the asymmetry as well as by the decoagulation. An underlying $S_{3}$-symmetry of the Hamiltonian was found and the relaxational modes seen in the hole functions could be understood from the general result.

The hierarchy of equations for these functions was considered and decoupled by a method introduced previously for the kinetic Ising model. In this way a complete solution was achieved. The decoupling approach lead to the same restrictions on the rates as Reshetikhin's integrability criterion. There are other solvable cases where a relation to the XXZ Hamiltonian is used [30. Whether further integrable situations exist, remains open.

As to the physical properties, it was found that the model has two phases with low and high average particle density, respectively. The corresponding density profiles and correlations were calculated. They contain the various lengths which arise from the interplay of decoagulation and asymmetry. The approach to the steady state also turns out to be interesting. Calculations show that at the transition point an initially full system first develops a plateau at the mean field value of the density before a slow relaxation to the linear density profile sets in. Only at the boundary one finds a simple algebraic decay with a $t^{-1 / 2}$ law.

A system with simple open ends, as considered here, is not the only possible case. More generally, one can supply and withdraw particles at the ends. Recent work has shown that, for pure hopping on the chain, this leads to interesting physical phenomena (boundary in-

duced transitions) and to new mathematical features (matrix-product states) [40, 41, 14, 42]. Since these processes are described by single spin operators at the ends of the chain, they can also be included in the present model. This is currently under investigation. Another possible direction would be to describe the coagulation process in more detail. This, however, would lead to models with more than two states per site which are more difficult to solve.

\section{Acknowledgements}

H. H. would like to thank the Deutsche Forschungsgemeinschaft for financial support. The authors also thank V. Rittenberg for many valuable discussions.

\section{References}

[1] Felderhof, B.U.: Rep. Math. Phys. 1, 219 (1970)

[2] Siggia, E.D.: Phys. Rev. B 16, 2319 (1977)

[3] Kimball, J.C.: J.Stat.Phys. 21, 289 (1979)

[4] Peschel, I., Emery, V.J.: Z. Phys. B 43, 241 (1981) 
[5] Glauber, R.J.: J. Math. Phys. 4, 241 (1963)

[6] Alexander, S., Holstein, T.: Phys Rev. B 18, 301 (1978)

[7] Dieterich, W., Fulde P., Peschel, I.: Adv. in Phys. 29, 527 (1980)

[8] Lushnikov, A.A.: Sov. Phys. JETP 64, 811 (1986) ;

Phys. Lett. A 120, 135 (1987)

[9] Alcaraz, F.C., Droz, M., Henkel, M., Rittenberg, V.: Ann. Phys. 230, 250 (1994)

[10] Alcaraz F.C., Rittenberg, V.: Phys. Lett. B 314, 377 (1993)

[11] Sandow, S., Schütz, G.: Europhys. Lett. 26, 7 (1994)

[12] Gwa, L.H., Spohn, H.: Phys. Rev. Lett. 68, 725 (1992) ;

Phys. Rev. A 46, 844 (1992)

[13] Henkel, M., Schütz, G.: Physica A 206, 187 (1994)

[14] Stinchcombe ,R.B., Schütz, G.: Europhys. Lett. 29, 1493 (1995)

[15] Sandow, S., Trimper, S.: Europhys. Lett. 21, 799 (1993)

Patzlaff, H., Trimper, S.: Phys. Lett. A 189, 187 (1994)

[16] Cardy, J.L.: Int. J. Mod. Phys. B8, 3463 (1994)

[17] Doering, C.R., ben-Avraham, D.: Phys. Rev. A 38, 3055 (1988)

[18] Burschka, M.A., Doering, C.R., ben-Avraham, D.: Phys. Rev. Lett. 63, 700 (1989)

[19] ben-Avraham, D., Burschka, M.A., Doering, C.R.: J. Stat. Phys. 60, 695 (1990)

[20] Doering, C.R., Burschka, M.A., Horsthemke, W.: J. Stat. Phys. 65, 953 (1991)

[21] Hinrichsen, H., Krebs, K., Pfannmüller, M., Wehefritz, B.: J. Stat. Phys. 78, 1429 (1995)

[22] Kroon, R., Fleurent ,H., Sprik, R.: Phys. Rev. E 47, 2462 (1993)

[23] Privman, V.: Phys. Rev. E 50, 50 (1994)

[24] Peschel, I., Rittenberg, V., Schultze, U.: Nucl. Phys. B 430, 633 (1994)

[25] Stey, G.C., Gusman, G.: J. Phys. C 6, 650 (1973)

[26] Saitoh, M.: J. Phys. C 6, 3255 (1973)

[27] Bedeaux, D., Schuler, K.E., Oppenheim, I.: J. Stat. Phys 2, 1 (1970)

[28] Kulish, P.P., Sklyanin, E.K.: in Lecture notes in physics Vol. 151, 61 Springer, Berlin (1982)

see also: Kennedy, T.: J. Phys. A 25, 2809 (1992) 
[29] Deker, U., Haake, F.: Z. Physik B 35, 281 (1979)

[30] Schütz, G.: J. Stat. Phys. 79, 243 (1995)

[31] Krug, J.: Phys. Rev. Lett. 67, 1882 (1991)

[32] Derrida, B., Domany, E., Mukamel, D.: J. Stat. Phys. 69, 667 (1992)

[33] Schütz, G., Domany, E.: J. Stat. Phys. 72, 277 (1992)

[34] Kadanoff, L.P., Swift, J.: Phys. Rev. 165, 310 (1968)

Doi, M.: J. Phys. A 9, 1465,1479 (1976)

Grassberger, P., Scheunert, M.: Fortschritte der Physik 28, 547 (1980)

[35] Lieb, E.H., Schultz, T.D., Mattis, D.C.: Ann. Phys. 16, 407 (1961)

[36] Baxter, R.J.: Exactly Solved Models in Statistical Mechanics, Academic Press, London (1982)

[37] Igloi, F., Turban, L., Peschel, I.: Adv. in Physics 42, 683 (1993)

[38] Hamermesh, M.: Group Theory Addison-Wesley, Reading (1962)

[39] Pokrovskii, V.L., Talapov, A.L.: Sov. Phys. JETP 51, 134 (1980)

[40] Derrida, B., Evans, M.R., Hakim, V., Pasquier, V.: J. Phys. A 26, 1493 (1993)

[41] Sandow, S.: Phys. Rev. E 50, 2664 (1994)

[42] Essler, F., Rittenberg, V.: Representations of the Quadric Algebra and Partially Asymmetric Diffusion with Open Boundaries con-mat/9506131(1995)

\section{Figure Captions:}

Fig. 1: Particle concentration $c(i)$ for $q=1.2$ and various values of $\Delta$ on a chain of 100 sites. 


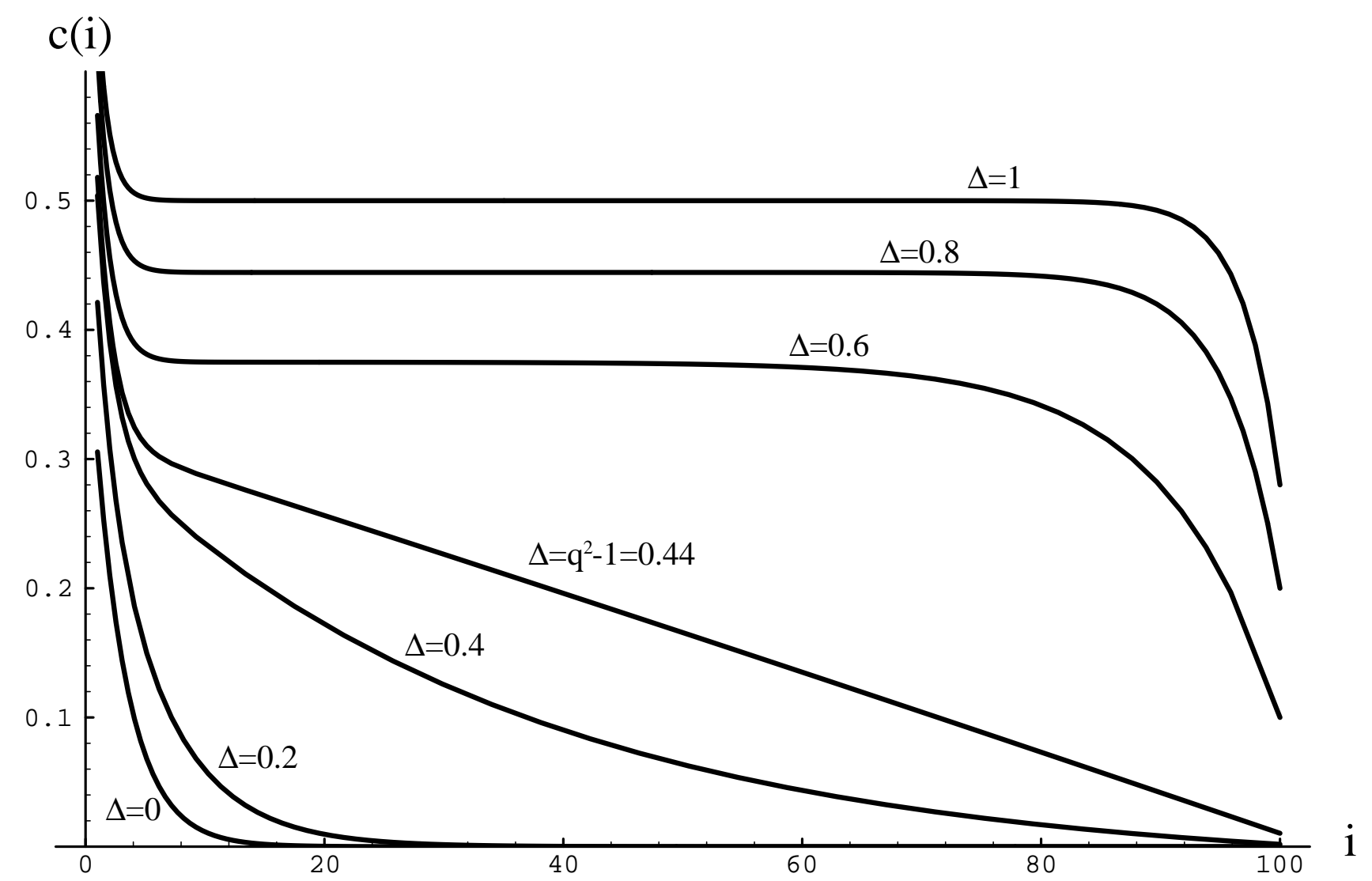

Figure 1 
